\title{
Shape sensitivity analysis of metallic nano particles
}

\author{
S. Sargheini and A. Paganini and R. Hiptmair and C. Hafner
}

Research Report No. 2016-15

March 2016

Seminar für Angewandte Mathematik

Eidgenössische Technische Hochschule

CH-8092 Zürich

Switzerland 


\title{
Shape sensitivity analysis of metallic nano particles
}

\author{
S. Sargheini*1, A. Paganini ${ }^{2}$, R. Hiptmair ${ }^{2}$, Ch. Hafner ${ }^{1}$ \\ ${ }^{1}$ Institute of Electromagnetic Fields, ETH Zurich, CH-8092 Zurich, Switzerland \\ 2 Seminar for Applied Mathematics, ETH Zurich, CH-8092 Zurich, Switzerland
}

\begin{abstract}
SUMMARY
Shape sensitivity measures the impact of small perturbations of geometric features of a problem on certain quantities of interest. The shape sensitivity of PDE constrained output functionals is derived with the help of shape gradients. In electromagnetic scattering problems, the standard procedure of the Lagrangian approach cannot be applied due to the fact that the solution of the state problem is complex valued. We derive a closed form formula of the shape gradient of a generic output functional constrained by Maxwell's equations. We employ cubic B-splines to model local deformations of the geometry, and derive sensitivity probings over the surface of the scatterer. We also define a sensitivity representative function over the surface of the scatterer based on local sensitivity measurements. Several numerical experiments are conducted to investigate the shape sensitivity of different output functionals for different geometric settings.

Copyright (c) 2010 John Wiley \& Sons, Ltd.
\end{abstract}

Received ...

KEY WORDS: Shape sensitivity analysis, Shape gradients, Maxwell's equations, Finite elements, Nano particles, Plasmonic.

\section{INTRODUCTION}

Over the last decades, plasmonic nano particles have attracted interest due to their localized field enhancement properties. The localized field can be exploited, e.g., for amplifying Raman and fluorescence scattering [1-4].

The effect of the shape and the size of nano particles on the performance of the device has been studied thoroughly in literature [5-7]. Most simulations investigate only the impact of a limited number of geometric parameters on the quantity of interest, whereas any change of shape can have a significant effect on the behavior of the structure. Since fabrication-based perturbations are inevitable, it is important to study the sensitivity of the performance of a structure with respect to small shape variations.

Shape sensitivity analysis studies how sensitive an output functional $\mathcal{J}$ is with respect to variations of the interface. To measure the shape sensitivity, one needs to evaluate the shape gradient of $\mathcal{J}$. In electromagnetic scattering problems, the shape functional measures a physical quantity of the problem. This means that $\mathcal{J}$ depends on the shape of the structure and is constrained by Maxwell's equations.

Derivation of shape gradients of PDE constraint shape functionals for scalar problems has been

*Correspondence to: Ramistrasse 101, CH-8092 Zurich, Switzerland. E-mail: saharss@ @ieh.ee.ethz.ch

Contract/grant sponsor: ETH Zurich; contract/grant number: CH1-02 11-1

Copyright (c) 2010 John Wiley \& Sons, Ltd.

Prepared using jnmauth.cls [Version: 2010/03/27 v2.00] 
studied thoroughly during the last decades [8-13]. In this paper, we investigate the shape sensitivity for 3D electromagnetic scattering. We extend the Lagrangian approach [8] to derive the shape gradient based on complex-valued solutions of adjoint $\mathbf{P}$ and state $\mathbf{E}$ boundary value problems. The shape sensitivity analysis for Maxwell's equations gets more complicated due to the regularity preservation [14, 15]. In Section 2, it is discussed that we need to use the covariant transformation in order to guarantee that solutions of adjoint and state problems in the mapped domain are still $H(\operatorname{curl} ; \Omega)$ functions. The final formula is in terms of a volume integral which is continuous in the energy norm and well-defined on the natural variational space.

We calculate the obtained shape gradient using perturbations with local support over the surface of the scatterer. Second order B-splines are used as probing perturbation fields. To get a representation of the shape sensitivity over the surface of the scatterer, we use the Hadamard-Zolesio structure theorem to define a representative function $g$ based on local shape gradients. We explicitly provide $g$ for different nano antennas with different shape functionals.

\section{SHAPE GRADIENT}

In this paper, we are mainly interested in $3 \mathrm{D}$ electromagnetic scattering. We assume the scatterer to occupy a bounded domain $\Omega_{s}$ with Lipschitz boundary $\partial \Omega_{s}$. We also introduce domain $\Omega$ with boundary $\partial \Omega$ such that $\Omega_{s} \subset \Omega:=\left\{\mathbf{r} \in \mathbb{R}^{3}:|\mathbf{r}|<R\right\}$, where $R>0$ (see Figure 1).

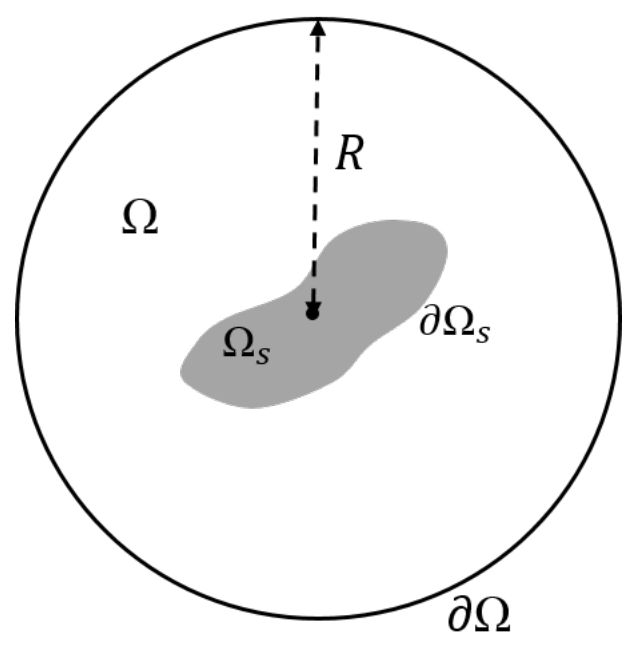

Figure 1. Domain definition for the scattering problem.

We assume that the permittivity of the material in both scatterer and surrounding domain $\epsilon(\mathbf{r}) \in \mathbb{C}$ is piecewise constant, linear, homogeneous, and isotropic. We also restrict ourselves to nonmagnetic materials $\left(\mu=\right.$ permeability of the free space $\left.\mu_{0}\right)$.

We define the space of three-dimensional vector functions as the following ${ }^{\dagger}$.

$$
H(\operatorname{curl}, \Omega)=\left\{\mathbf{u} \in L^{2}(\Omega) \mid \nabla \times \mathbf{u} \in L^{2}(\Omega)\right\}
$$

\footnotetext{
${ }^{\dagger}$ For the sake of readability, we use the same notation for scalar and vectorial Sobolev spaces.
} 
The time harmonic electric field $\mathbf{E} \in H(\operatorname{curl}, \Omega)$ at angular frequency $\omega$ is obtained as the solution to the following weak formulation of the second order Maxwell's equation [16, Sec. 4.1]

$$
\begin{array}{ll}
\int_{\Omega} \nabla \times \mathbf{E} \cdot \nabla \times \overline{\mathbf{W}}-k^{2}(\mathbf{r}) \mathbf{E} \cdot \overline{\mathbf{W}} d \mathbf{r}-\int_{\partial \Omega} \operatorname{DtN}[\mathbf{E}] \cdot \overline{\mathbf{W}} d s(\mathbf{r})= & \\
\int_{\partial \Omega}\left(\left(\nabla \times \mathbf{E}_{i}\right) \times \hat{n}\right) \cdot \overline{\mathbf{W}}-\operatorname{DtN}\left[\mathbf{E}_{i}\right] \cdot \overline{\mathbf{W}} d s(\mathbf{r}) & \forall \mathbf{W} \in H(\operatorname{curl}, \Omega),
\end{array}
$$

where $\mathbf{E}_{i}$ is the incident electric field, and $k(\mathbf{r}):=w \sqrt{\epsilon(\mathbf{r}) \mu_{0}}$ is the wavenumber. By DtN we denote Dirichlet-to-Neumann operator (also named Calderon map) which is used to bound the solution domain [16, Ch. 9, Sec. 4]. $\overline{\mathbf{W}}$ is the complex conjugate of $\mathbf{W}$.

To describe physical outputs based on the solution of (1), we rely on shape functionals of the form

$$
\mathcal{J}(\Omega):=\int_{\Omega_{m}} j(\mathbf{E}) d \mathbf{r}
$$

where $j: \mathbb{C}^{3} \rightarrow \mathbb{R}$, and $\Omega_{m}$ is the measurement region.

The sensitivity of $\mathcal{J}$ with respect to perturbations of the surface of the scatterer can be expressed through the Eulerian derivative of $\mathcal{J}$ in the direction of the vector field $\mathcal{V} \in C^{1}(\Omega)$ [8, Ch. 1, Sec. 9]

$$
d \mathcal{J}(\Omega ; \mathcal{V}):=\lim _{s \searrow 0} \frac{\mathcal{J}\left(T_{s \cdot \mathcal{V}}(\Omega)\right)-\mathcal{J}(\Omega)}{s},
$$

where the mapping $T_{\mathcal{V}}$ models domain perturbations in the direction $\mathcal{V}$

$$
T_{\mathcal{V}}(\mathbf{r}):\left\{\begin{aligned}
\mathbb{R}^{3} & \rightarrow \mathbb{R}^{3} \\
\mathbf{r} & \rightarrow \mathbf{r}+\mathcal{V}(\mathbf{r})
\end{aligned}\right.
$$

In literature, $d \mathcal{J}$ is called the shape gradient of $\mathcal{J}$ at $\Omega$ [9].

To calculate the shape gradient of the output functional (2), we first need to make the following assumption and also to state Lemmas 1 and 2.

\section{Assumption 1}

We assume that the perturbation $\mathcal{V} \in C^{1}(\Omega)$ and it vanishes on $\partial \Omega$ and inside $\Omega_{m}$ the measurement region.

\section{Lemma 1}

The covariant transformations

$$
\begin{aligned}
& \Psi_{\mathcal{V}}:\left\{\begin{aligned}
H\left(\operatorname{curl} ; T_{\mathcal{V}}(\Omega)\right) & \mapsto H(\operatorname{curl} ; \Omega) \\
\mathbf{F} & \mapsto D T_{\mathcal{V}}^{T}\left(\mathbf{F} \circ T_{\mathcal{V}}\right)
\end{aligned}\right. \\
& \Phi_{\mathcal{V}}:\left\{\begin{array}{rl}
H\left(\operatorname{div} ; T_{\mathcal{V}}(\Omega)\right) & \mapsto H(\operatorname{div} ; \Omega) \\
\mathbf{F} & \mapsto \operatorname{det} D T_{\mathcal{V}}\left(D T_{\mathcal{V}}^{-1}\left(\mathbf{F} \circ T_{\mathcal{V}}\right)\right)
\end{array},\right. \\
& \text { and satisfy } \nabla \times \Psi_{\mathcal{V}}(\mathbf{F})=\Phi_{\mathcal{V}}\left(\nabla^{\mathcal{V}} \times \mathbf{F}\right),
\end{aligned}
$$

are isomorphism [17]. By $\nabla^{\mathcal{V}}$ we denote the derivative in the transformed domain. 


\section{Lemma 2}

Let $Y(\Omega)$ be a vector space over $\mathbb{C}$ and $u \in Y(\Omega)$ be the solution to the following problem

$$
\operatorname{Re}\{a(u, v)-\ell(v)\}=0 \quad \forall v \in Y(\Omega),
$$

where $a: Y \times Y \mapsto \mathbb{C}$ and $\ell: Y \mapsto \mathbb{C}$ are sesquilinear and linear forms defined on $Y(\Omega)$. Then $u$ is the solution of the following variational problem

$$
a(u, v)-\ell(v)=0 \quad \forall v \in Y(\Omega),
$$

Proof

If $\arg (a(u, v)-\ell(v))=\varphi$, then

$$
\begin{aligned}
e^{-i \varphi}(a(u, v)-\ell(v)) & =a\left(u, e^{-i \varphi} v\right)-\ell\left(e^{-i \varphi} v\right) \\
& =\operatorname{Re}\left\{a\left(u, e^{-i \varphi} v\right)-\ell\left(e^{-i \varphi} v\right)\right\} \\
& =0 .
\end{aligned}
$$

\section{Theorem 1}

The shape gradient of the shape functional $\mathcal{J}(\Omega)$ (2) subject to Maxwell's equation (1) is

$$
\begin{gathered}
d \mathcal{J}=\operatorname{Re}\left(\int_{\Omega} \nabla \times \mathbf{E} \cdot\left(D \mathcal{V}+D \mathcal{V}^{\top}\right) \nabla \times \overline{\mathbf{P}}+\mathbf{E} \cdot\left(D \mathcal{V}+D \mathcal{V}^{\top}\right) \overline{\mathbf{P}}\right. \\
\left.-\nabla \cdot \mathcal{V}\left(\nabla \times \mathbf{E} \cdot \nabla \times \overline{\mathbf{P}}+k^{2}(\mathbf{r}) \mathbf{E} \cdot \overline{\mathbf{P}}\right) d \mathbf{r}\right)
\end{gathered}
$$

where $D \mathcal{V}$ is the Jacobian of $\mathcal{V}$, and $\mathbf{P}(\mathbf{r}) \in H(\operatorname{curl}, \Omega)$ is the solution of the following variational problem

$$
\begin{array}{rlrl}
\int_{\Omega} \nabla \times \overline{\mathbf{P}} & \nabla \times \mathbf{W}-k^{2}(\mathbf{r}) \overline{\mathbf{P}} \cdot \mathbf{W} d \mathbf{r}-\int_{\partial \Omega} \overline{\operatorname{DtN}^{*}[\mathbf{P}]} \cdot \mathbf{W} d s(\mathbf{r}) & \\
=\int_{\Omega_{m}} \overline{j^{\prime}}(\mathbf{E}) \cdot \mathbf{W} d \mathbf{r} & \forall \mathbf{W} \in H(\operatorname{curl} ; \Omega) .
\end{array}
$$

By $j^{\prime}$, we denote the derivative of $j$. The adjoint operator $D t N^{*}$ is defined as $\langle\operatorname{DtN}[\mathbf{A}], \mathbf{B}\rangle_{\partial \Omega}=$ $\left\langle\mathbf{A}, \operatorname{DtN}^{*}[\mathbf{B}]\right\rangle_{\partial \Omega}$, where $\mathbf{A}, \mathbf{B} \in H(\operatorname{curl}, \Omega)$ and $\langle\mathbf{A}, \mathbf{B}\rangle_{\partial \Omega}=\int_{\partial \Omega} \mathbf{A} \cdot \mathbf{B} d s(\mathbf{r})$.

Proof

Since the shape functional (2) is real valued while the constraint (1) is complex valued, the standard Lagrangian approach [8, Ch. 10, Sec. 5] cannot be applied. Following [18], we define the Lagrangian as

$$
\begin{aligned}
\mathscr{L}(\Omega, \mathbf{U}, \mathbf{P})=\operatorname{Re} & \left\{\mathcal{J}(\mathbf{U})+\int_{\Omega} \nabla \times \mathbf{U} \cdot \nabla \times \overline{\mathbf{P}}-k^{2}(\mathbf{r}) \mathbf{U} \cdot \overline{\mathbf{P}} d \mathbf{r}\right. \\
& \left.-\int_{\partial \Omega} \operatorname{DtN}[\mathbf{U}] \cdot \overline{\mathbf{P}}-\operatorname{DtN}\left[\mathbf{E}_{i}\right] \cdot \overline{\mathbf{P}}+\left(\left(\nabla \times \mathbf{E}_{i}\right) \times \hat{n}\right) \cdot \overline{\mathbf{P}} d s(\mathbf{r})\right\},
\end{aligned}
$$

where the functions $\mathbf{U}$ and $\mathbf{P}$ are in $H(\operatorname{curl} ; \Omega)$.

Inserting $\mathbf{U}=\mathbf{E}(\Omega)$ gives

$$
\mathcal{J}(\Omega)=\mathscr{L}(\Omega, \mathbf{E}(\Omega), \mathbf{P}) \quad \forall \mathbf{P} \in H(\operatorname{curl}, \Omega) .
$$


Consequently, we have

$$
d \mathcal{J}(\Omega, \mathcal{V})=\left\langle\frac{\partial \mathscr{L}(\Omega, \mathbf{E}(\Omega), \mathbf{P})}{\partial \mathbf{E}}, \mathbf{E}^{\prime}(\Omega, \mathcal{V})\right\rangle+\left\langle\frac{\partial \mathscr{L}(\Omega, \mathbf{E}(\Omega), \mathbf{P})}{\partial \Omega}, \mathcal{V}\right\rangle,
$$

where $\mathbf{E}^{\prime}(\Omega, \mathcal{V})$ denotes the derivative of $\mathbf{E}(\Omega)$ with respect to $\Omega$ in the direction $\mathcal{V}$. Since $\mathbf{P}$ is an arbitrary parameter, we choose $\mathbf{P} \in H(\operatorname{curl}, \Omega)$ such that

$$
\left\langle\frac{\partial \mathscr{L}(\Omega, \mathbf{E}(\Omega), \mathbf{P})}{\partial \mathbf{E}}, \mathbf{W}\right\rangle=0 \quad \forall \mathbf{W} \in H(\operatorname{curl}, \Omega),
$$

which leads to the following adjoint problem

$$
\begin{array}{ccc}
\operatorname{Re}\left\{\int_{\Omega} \nabla \times \overline{\mathbf{P}} \cdot \nabla \times \mathbf{W}-k^{2}(\mathbf{r}) \overline{\mathbf{P}} \cdot \mathbf{W} d \mathbf{r}-\int_{\partial \Omega} \overline{\operatorname{DtN}^{*}[\mathbf{P}]} \cdot \mathbf{W} d s(\mathbf{r})\right. \\
\left.-\int_{\Omega} \overline{j^{\prime}}(\mathbf{U}) \cdot \mathbf{W} d \mathbf{r}\right\}=0 & \forall \mathbf{W} \in H(\operatorname{curl}, \Omega) .
\end{array}
$$

As implied by Lemmma 2, taking the real part in (12) is redundant . Hence, one can simply dispose Re in (12) to derive the final adjoint formulation in (8).

By (10) and (11), we can write that

$$
d \mathcal{J}(\Omega, \mathcal{V})=\left\langle\frac{\partial \mathscr{L}(\Omega, \mathbf{E}(\Omega), \mathbf{P})}{\partial \Omega}, \mathcal{V}\right\rangle
$$

Based on Lemma 1, the Lagrangian in the transformed domain is

$$
\begin{aligned}
\mathscr{L}\left(T_{s \cdot \mathcal{V}}(\Omega), \mathbf{E}, \mathbf{P}\right)=\operatorname{Re} & \left\{\mathcal{J}(\mathbf{E})+\int_{T_{s \cdot \mathcal{V}}(\Omega)} \nabla \times \mathbf{E} \cdot \nabla \times \overline{\mathbf{P}}-k^{2}(\mathbf{r}) \mathbf{E} \cdot \overline{\mathbf{P}} d \mathbf{r}\right. \\
& \left.-\int_{\partial \Omega} \operatorname{DtN}[\mathbf{E}] \cdot \overline{\mathbf{P}}-\operatorname{DtN}\left[\mathbf{E}_{i}\right] \cdot \overline{\mathbf{P}}+\left(\left(\nabla \times \mathbf{E}_{i}\right) \times \hat{n}\right) \cdot \overline{\mathbf{P}} d s(\mathbf{r})\right\} \\
=\operatorname{Re} & \left\{\mathcal{J}\left(\mathbf{E}^{s}\right)+\int_{\Omega} \nabla \times \mathbf{E}^{s} \cdot \frac{D T_{s \cdot \mathcal{V}}}{\operatorname{det} D T_{s \cdot \mathcal{V}}} \frac{D T_{s \cdot \mathcal{V}}^{T}}{\operatorname{det} D T_{s \cdot \mathcal{V}}} \nabla \times \overline{\mathbf{P}}^{s}\left|\operatorname{det} D T_{s \cdot \mathcal{V}}\right| d \mathbf{r}\right. \\
& -\int_{\Omega} k^{2}(\mathbf{r}) \mathbf{E}^{s} \cdot D T_{s \cdot \mathcal{V}}^{-T} D T_{s \cdot \mathcal{V}}^{-1} \overline{\mathbf{P}}^{s}\left|\operatorname{det} D T_{s \cdot \mathcal{V}}\right| d \mathbf{r} \\
& \left.-\int_{\partial \Omega} \operatorname{DtN}\left[\mathbf{E}^{s}\right] \cdot \overline{\mathbf{P}}^{s}-\operatorname{DtN}\left[\mathbf{E}_{i}\right] \cdot \overline{\mathbf{P}}^{s}+\left(\left(\nabla \times \mathbf{E}_{i}\right) \times \hat{n}\right) \cdot \overline{\mathbf{P}}^{s} d s(\mathbf{r})\right\},
\end{aligned}
$$

where $\mathbf{F}^{s}=\Psi_{s \cdot \mathcal{V}}(\mathbf{F})$.

Using Tailor expansion, one can simply show that [9, Ch. 2]

$$
\left\{\begin{aligned}
\left(\operatorname{det} D T_{s \cdot \mathcal{V}}\right)^{-1} & =1-s \nabla \cdot \mathcal{V}+O\left(s^{2}\right) \\
D T_{s \cdot \mathcal{V}}^{-1} & =\mathcal{I}-s D \mathcal{V}+O\left(s^{2}\right)
\end{aligned} \quad \text { for } s \rightarrow 0,\right.
$$

where $\mathcal{I}$ is the identity matrix.

By plugging (15) in (14), one can derive the corresponding formula for the shape gradient of $\mathcal{J}$ in (7). 


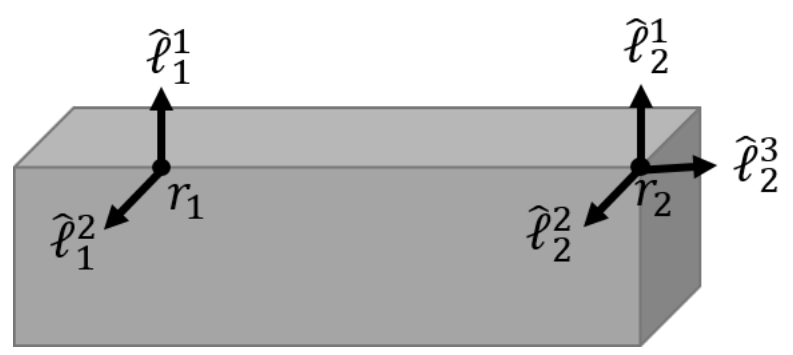

Figure 2. Directions of B-splines assigned to $\mathbf{r}_{1}$ and $\mathbf{r}_{2}$ located on an edge and on a corner, respectively.

\section{SHAPE SENSITIVITY REPRESENTATIVE AND NUMERICAL APPROXIMATION}

Our aim is to define a representative function $g$ of $d \mathcal{J}$ on the boundary $\partial \Omega_{s}$. To justify the existence of $g$, we make the following assumption.

\section{Assumption 2}

We assume that $d \mathcal{J}: \mathcal{V} \rightarrow d \mathcal{J}(\Omega, \mathcal{V})$ defines a linear continuous operator on $H^{1}(\Omega)$.

\section{Remark 1}

Assumption 2 is fulfilled if, for instance, $\mathbf{E}, \mathbf{P} \in\left\{\mathbf{u} \in L^{4}(\Omega) \mid \nabla \times \mathbf{u} \in L^{4}(\Omega)\right\}$.

For a smooth $\partial \Omega_{s}$, the Hadamard-Zolesio structure theorem [8, Ch. 9, Thm 3.6] and Assumption 2 guarantee the existence of a function $g \in H^{-1 / 2}\left(\partial \Omega_{s}\right)$ such that

$$
\int_{\partial \Omega_{s}}(\mathcal{V}(\mathbf{r}) \cdot \hat{n}(\mathbf{r})) g(\mathbf{r}) d s(\mathbf{r})=d \mathcal{J}(\Omega ; \mathcal{V}) \quad \forall \mathcal{V} \in H^{1}(\Omega) .
$$

Note that, if the output functional measures the energy flux, then the dimension of $g$ is $\left[\frac{\mathrm{J}}{\mathrm{m}^{4}}\right]$.

The absolute value of the shape sensitivity representative function $|g|$ shows how the output functional changes under the influence of local small shape perturbations normal to the surface of the scatterer. Based on the values of $|g|$ over $\partial \Omega_{s}$, one can see which parts of the structure must be fabricated with higher accuracy.

To compute $g$ numerically, we consider only finitely many vector fields $V_{n}=\left\{\mathcal{V}_{i}\right\}_{i=1}^{n}$, where $n:=\operatorname{dim} V_{n}$. More specifically, we consider probing vector fields of the following form

$$
\mathcal{V}_{i}=B_{i} \hat{\ell}_{i}
$$

where $B_{i}$ is a scalar tensor product B-spline [19, Sec. 7.3] of degree 2 centered on $\mathbf{r}_{i} \in \partial \Omega_{s}$, and $\hat{\ell}_{i}$ is a unit vector denoting the direction of $\mathcal{V}_{i}$.

$\mathrm{B}$-splines are piecewise polynomials and have compact support. To construct these functions we consider a regular grid on the surface of the scatterer. The $i$ th B-spline $B_{i}$ is centered on the knot $\mathbf{r}_{i}$ of this grid. In light of (16), the direction $\hat{\ell}_{i}$ is chosen to be normal to $\partial \Omega_{s}$ at the point $\mathbf{r}_{i}$.

\section{Remark 2}

The normal direction may not be uniquely defined if the boundary is piecewise smooth only, for instance for polyhedral domains. In the presence of edges and corners, one needs to consider all the possible directions for the shape perturbation. As shown in Figure 2, we assign more than one probing vector field $\left\{B_{i} \hat{\ell}_{i}^{j}\right\}_{j=1}^{q}$ for perturbations which are centered on edges or corners. The number of vector fields $q$ is equal to the number of faces that share the given edge or corner. By $\hat{\ell}_{i}^{j}$, we denote the normal direction on the $j$-th face sharing the corresponding edge or corner.

To solve (16), we discretize the function space of $g$ and define $g_{h} \in Y_{h}$ as follows

$$
g_{h}=\sum_{j=1}^{m} \tilde{g}_{j} b_{j}(\mathbf{r}),
$$


where $\tilde{g}_{j} \in \mathbb{R}$, and $b_{j} \in Y_{h}$ are basis functions of $Y_{h}, j=1, \ldots, m$, with $m:=\operatorname{dim} Y_{h}$.

Using (16) and (17), we have

$$
\tilde{\mathbf{A}} \mathbf{g}=\mathbf{f},
$$

where $\mathbf{g}:=\left(\tilde{g}_{1}, \cdots, \tilde{g}_{m}\right)^{\top}$ and $\mathbf{f}:=\left(d \mathcal{J}\left(D ; \mathcal{V}_{1}\right), \cdots, d \mathcal{J}\left(D ; \mathcal{V}_{n}\right)\right)^{\top}$. The entries of the $n \times m$ matrix $\tilde{\mathbf{A}}$ are given by

$$
\tilde{\mathbf{A}}_{i j}=\int_{\partial \Omega_{s}}\left(\mathcal{V}_{i} \cdot \hat{n}\right) b_{j} d s(\mathbf{r}) .
$$

In order for $g_{h}$ to be sufficiently accurate, the trial space $Y_{h}$ must be large enough, we impose at least $m>n$. In this case, the system of linear equations in (18) is underdetermined. Using the least squares method, we define

$$
\tilde{X}:=\underset{\mathbf{x} \in \mathbb{R}^{m}}{\operatorname{argmin}}\|\tilde{\mathbf{A}} \mathbf{x}-\mathbf{f}\|_{\mathbb{R}^{n}} .
$$

We must mention that $\tilde{X}$ is a set which can contain more than one element. To obtain a unique result, we use the $H^{1}$-regularization and define $\mathrm{g}$ as an element of $\tilde{X}$ which has the minimum norm

$$
\mathbf{g}=\underset{\mathbf{x} \in \tilde{X}}{\operatorname{argmin}}\|\mathbf{x}\|_{\mathbf{H}}^{2},
$$

where $\|\mathbf{x}\|_{\mathbf{H}}=\mathbf{x}^{\top} \mathbf{H} \mathbf{x}$. By $\mathbf{H}$ we denote a $m \times m$ matrix with $\mathbf{H}_{i j}=\int_{\partial \Omega_{s}} \nabla_{t} u_{i} \cdot \nabla_{t} b_{j} d s(\mathbf{r})$, where $\nabla_{t}$ is the tangential gradient on the surface $\partial \Omega_{s}$. Note that to compute $g$ we must make the trial space $Y_{h}$ satisfy $Y_{h} \subset H^{1}\left(\partial \Omega_{s}\right)$.

\section{Remark 3}

The choice of $H^{1}$-seminorm in (21) cannot be justified rigorously. However, we experienced that employing the $L^{2}$-regularization creates artifacts. Employing higher order regularizations might be too strong a constraint.

\section{NUMERICAL RESULTS}

We solve the variational problems (1) and (8) numerically. We use third order Nedelec finite elements on a quasi-uniform tetrahedral $\mathrm{mesh}^{\ddagger}$. To truncate the solution domain and approximate the DtN map, we use a box Perfectly Matched Layer (PML). The integrals in the domain are computed by a 6th order quadrature rule in each element. We also use first order finite elements to approximate $g$ over the surface of scatterers.

To simplify implementations, we locate probing vector fields on vertices of a grid which is independent of the FEM mesh. The size of the B-spline grid must be fine enough to model surface perturbations properly. In our simulations, we observe that $h_{B}=3 \max \left(h_{\mathrm{FEM}}\right)$ is a good choice ( $h_{B}$ and $h_{\mathrm{FEM}}$ are element sizes of the B-spline grid and the FEM mesh, respectively).

In numerical experiments, we consider two types of output functionals. The first type measures the near field data within the domain $\Omega_{m} \subset \Omega$

$$
\mathcal{J}^{\text {near }}=\int_{\Omega_{m}}\left|\mathbf{E}_{s}\right|^{2} d \mathbf{r}
$$

where $\mathbf{E}_{s}=\mathbf{E}-\mathbf{E}_{i}$ is the scattered field.

As a second type of the output functional, we consider the field value at far distances which is

\footnotetext{
$\mp$ Our experiments are based on the finite element library NGSolve developed by Joachim Schoeberl at the Vienna University of Technology.

http://sourceforge.net/projects/ngsolve.
} 


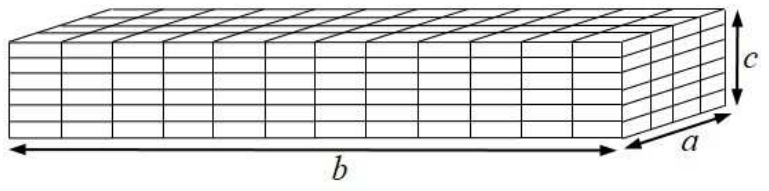

(a)

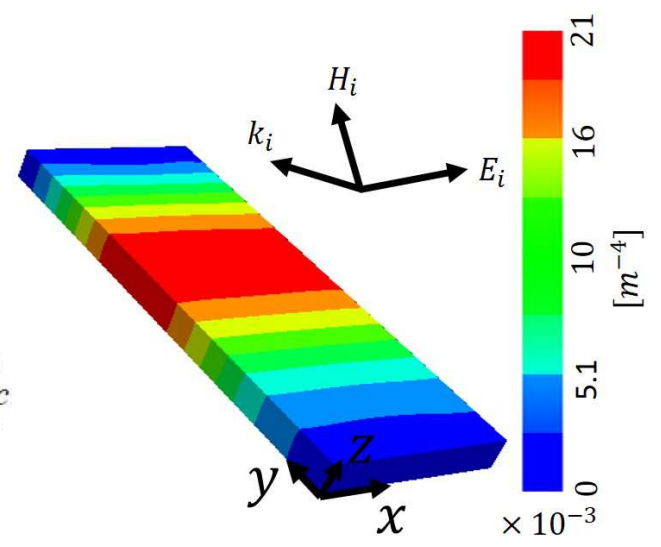

(b)

Figure 3. (a) The silver brick with dimensions $(a, b, c)=(50,150,10) \mathrm{nm}$. The substrate material is glass with refractive index $n_{2}=1.5$. (b) The relative sensitivity representative $\left|\frac{g}{\mathcal{J}}\right|$ obtained based on finite element approximation of $\mathbf{E}$ and $\mathbf{P}$ using 469405 DoFs and considering 1116 B-splines as local perturbations. The output functional measures the energy flux of the far-field.

collected through an objective lens with the opening angle of $\alpha=74^{\circ}$ and centered on the $z$-axis

$$
\mathcal{J}^{\text {far }}=\int_{\Omega_{\text {lens }}}\left|\mathbf{E}_{\infty}\right|^{2} d \mathbf{r}
$$

where $\mathbf{E}_{\infty}$ is the far field [20, Eq. 4.10]. By $\Omega_{\text {lens }}$, we denote the area of the objective lens.

\subsection{Silver brick}

In the first experiment, we investigate the sensitivity of the energy flux at far field (23) with respect to shape perturbations over the surface of a silver brick (see Figure 3a). The grid over the surface of the scatterer in Figure 3a represents the B-spline grid which in this example consists of 1116 vector fields. The excitation wavelength is $\lambda=500 \mathrm{~nm}$ and the incident wave is $\mathbf{E}_{i}=\exp \left(i k_{0}\left(\sin \left(\frac{\pi}{3}\right) x-\cos \left(\frac{\pi}{3}\right) z\right)\right) \hat{y}$ (where $\hat{y}$ is the unit vector in the direction $y$ ). Optical constants of the silver at the given frequency is obtained using the data provided by [21]. The nano-particle is mounted on a glass substrate with $n_{2}=1.5$.

We approximate the solutions of state and adjoint problems by using the finite element method with 469405 degrees of freedom (DoFs). The shape sensitivity representative function $g$ is also obtained by considering a finite element mesh over the surface of the brick with 3646 DoFs.

Figure $3 \mathrm{~b}$ shows the absolute value of the relative shape sensitivity $\left|\frac{g}{\mathcal{J}}\right|$ over the scatterer. Based on the obtained result, the far-field pattern is mostly sensitive to the central part of the brick.

To see the effect of the finite element mesh on the sensitivity pattern, we repeat the previous experiment using two finer meshes to achieve 886890 and 1163595 DoFs for discretizing state and adjoint problems, while all the other settings are kept untouched. As shown in Figures $4 \mathrm{a}$ and $4 \mathrm{~b}$, the sensitivity representative is not affected significantly by the mesh resolution.

Finally, we investigate the effect of the B-spline grid resolution on $g$ by increasing the number of probation vector fields to 2126 while the finite element method used to solve state and adjoint problems have 469405 DoFs. Based on the result shown in Figure 4c, the sensitivity pattern remains almost the same when increasing the number of B-splines.

\subsection{Groove antenna}

We now repeat the previous experiment by adding a V-groove in the central part of the silver brick (see Figure 5a) [22]. The dimension of the nano-particle is $(a, b, c)=(50,150,10) \mathrm{nm}$ and 


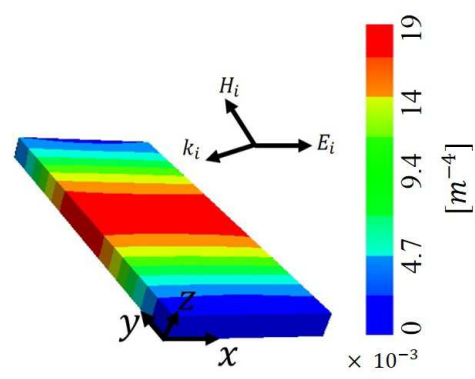

(a)

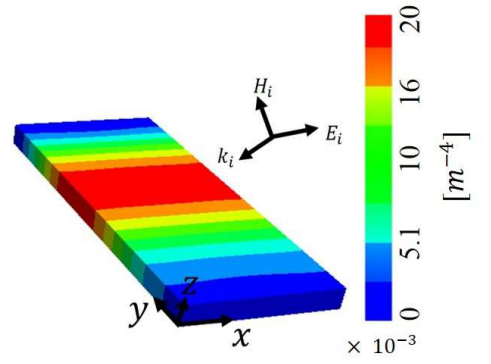

(b)

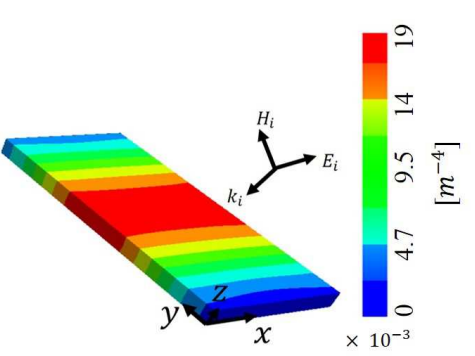

(c)

Figure 4. The relative sensitivity representative $\left|\frac{g}{\mathcal{J}}\right|$ over the silver brick obtained based on (a) finite element approximation of $\mathbf{E}$ and $\mathbf{P}$ using 886890 DoFs and considering 1116 B-splines as local perturbations, (b) finite element approximation of $\mathbf{E}$ and $\mathbf{P}$ using 1163595 DoFs and considering $1116 \mathrm{~B}$-splines as local perturbations, and (c) finite element approximation of $\mathbf{E}$ and $\mathbf{P}$ using 469405 DoFs and considering 2126 B-splines as local perturbations.

$\left(d_{1}, d_{2}, d_{3}\right)=(6,6,6) \mathrm{nm}$. Material properties and the excitation are similar to those in the previous experiment. The finite element method used to solve Maxwell's equations employs 901860 DoFs.

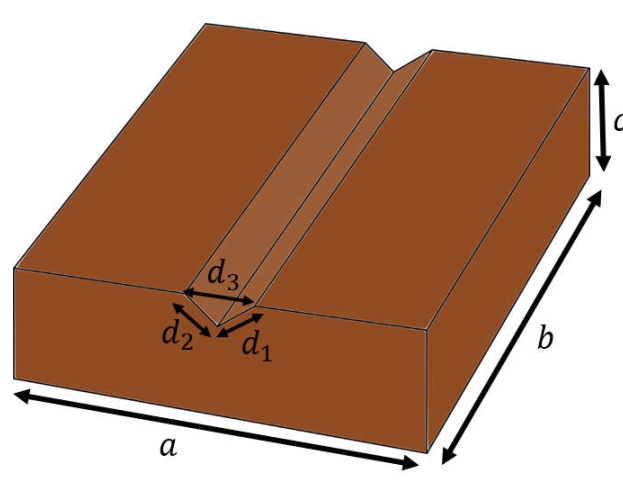

(a)

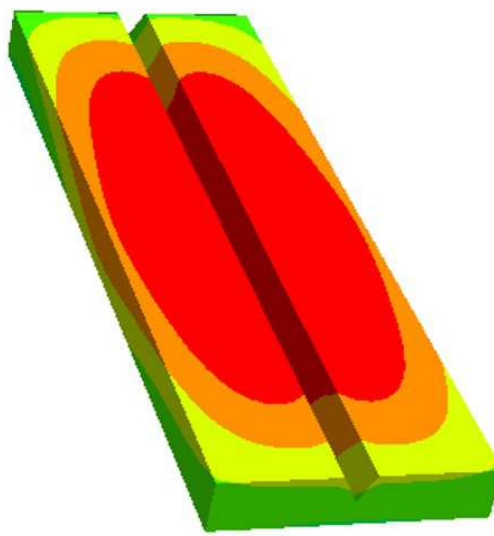

(b)

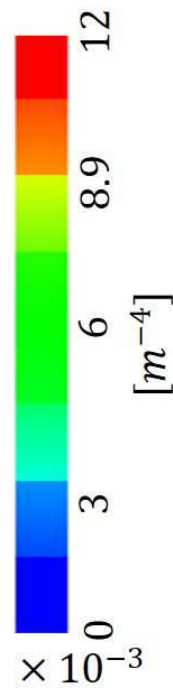

Figure 5. (a) The silver V-groove waveguide. The dimensions are $(a, b, c)=(50,150,10) \mathrm{nm}$ and $\left(d_{1}, d_{2}, d_{3}\right)=(6,6,6) \mathrm{nm}$. The substrate material is glass with refractive index $n_{2}=1.5$. (b) The relative sensitivity representative $\left|\frac{g}{\mathcal{J}}\right|$ over the V-groove. The output functional measures the energy flux of the far-field.

The experiment investigates the sensitivity of the far-field functional (23). The B-spline grid consists of $1225 \mathrm{~B}$-splines over the surface of the groove $\partial \Omega_{s}$. To discretize $g$ over $\partial \Omega_{s}$, we exploit a finite element discretization with 3862 DoFs.

As shown in Figure 5b, the energy in the far field is more sensitive to the shape of the channel. Comparing Figure $5 b$ and Figure $3 b$, one can see that the addition of the V-groove channel changes the sensitivity profile such that $\left|\frac{g}{\mathcal{J}}\right|$ is no more concentrated in the central part of the structure. It can be explained by considering the waveguiding effect of the V-groove based on Channel Plasmon 
Polariton (CPP) effect [22]. One other observation based on Figure 5b is that the end parts of the channel have less effect on the far-field pattern.

\subsection{Nano antenna consisting two rectangular blocks in free space}

In this experiment, we consider a gold nano antenna made of two rectangular gold blocks, brick-1 and brick-2 located in free space (see Figure 6a).

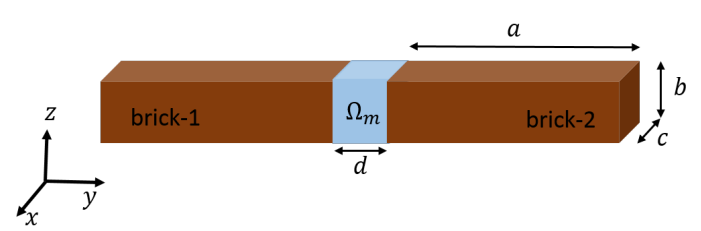

(a)

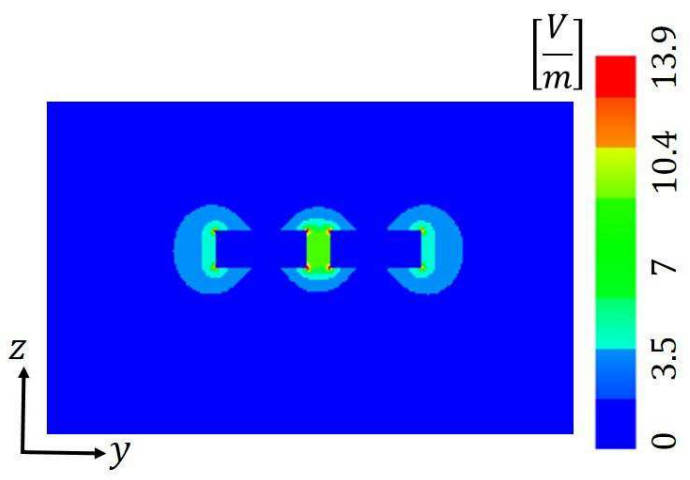

(b)

Figure 6. (a) Gold nano antenna consisting of two brown rectangular-blocks with dimensions $(a, b, c, d)=$ $(98.5,40,40,25) \mathrm{nm}$. The blue domain in the gap area $\Omega_{m}$ shows the near-field integration region. (b) The absolute value of the electric field at $\lambda=640 \mathrm{~nm}$.

In our simulations we consider $\lambda=770 \mathrm{~nm}$, and $\mathbf{E}_{i}=\exp \left(i k_{0}\left(\sin \left(\frac{\pi}{3}\right) x-\cos \left(\frac{\pi}{3}\right) z\right)\right) \hat{y}$. Since gold is a dispersive material, we use the measured data provided by [21] to obtain optical constants of gold at a given frequency. The finite element method used to solve Maxwell's equations employs 604595 DoFs. Since the structure is symmetric, we perform the sensitivity analysis only on one of the arms of the antenna, brick-1.

The B-spline grid exploits 1166 B-splines over the surface of brick-1, and the finite element mesh used to discretize $g$ has 1811 DoFs.

We solved the problem for both near-field and far-field functionals. The near-field integration region $\Omega_{m}$ in Equation (22) is shown as a region in light blue in Figure 6a. Relative sensitivities of $\mathcal{J}^{\text {near }}$ and $\mathcal{J}^{\text {far }}$ over brick-1 are shown in Figures $7 \mathrm{a}$ and $7 \mathrm{~b}$, respectively. As one can see, both near-field and far-field functionals are the most sensitive to changes in the left side of brick- 1 where is closer to the gap. An interesting observation is that the far-field functional, unlike the near-field one, is less sensitive to perturbations of edges and corners. This means that despite the high field localization around corners, they do not have significant effect on the far-field pattern, whereas the near-field is strongly affected by their shape.

\subsection{Nano antenna consisting two rectangular blocks over a substrate}

To see the effect of a substrate on $g$, we mount the antenna consisting of two rectangular blocks on a glass substrate located at $z<0$ with the refractive index $n_{2}=1.5$ (see Figure 8a). The size of the antenna, the wavelength, and the incident field are the same as those in the previous experiment. Let $\Gamma_{s}$ be the face of brick-1 which touches the substrate. Probing functions located on $\Gamma_{s}$ perturb the surface of the substrate as well. This means that the part of the substrate surface which is touching the antenna is no longer flat after deformations.

The sensitivity representative function over the surface of brick-1 for the far-field functional is shown in Figure 8b. As one can see, the presence of the substrate causes $g$ to increase on $\Gamma_{s}$. In 


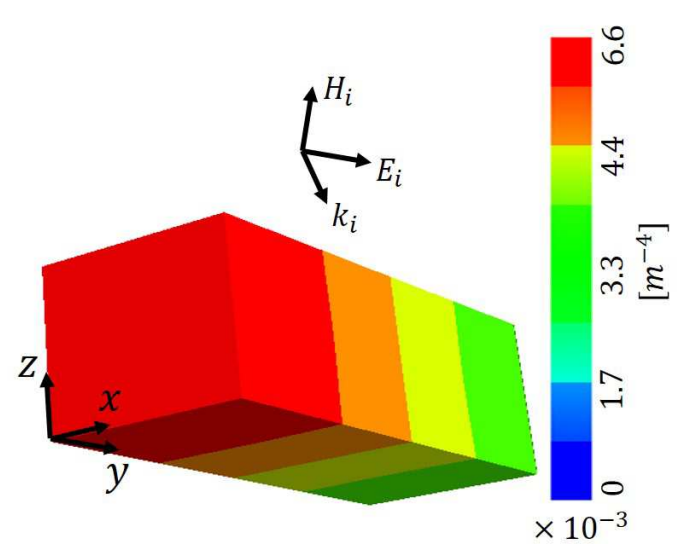

(a)

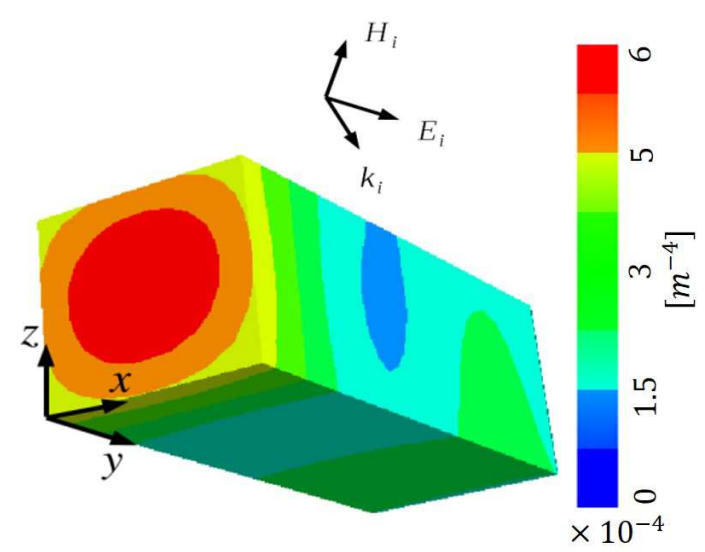

(b)

Figure 7. The relative sensitivity representative $\left|\frac{g}{\mathcal{J}}\right|$ of (a) near-field (b) far-field functionals over the right arm of the gold nano antenna consisting of two rectangular blocks.

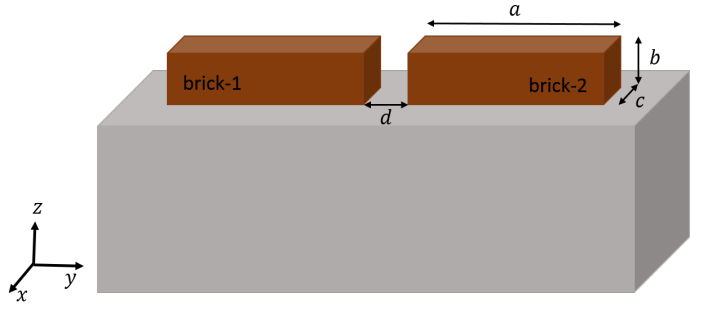

(a)

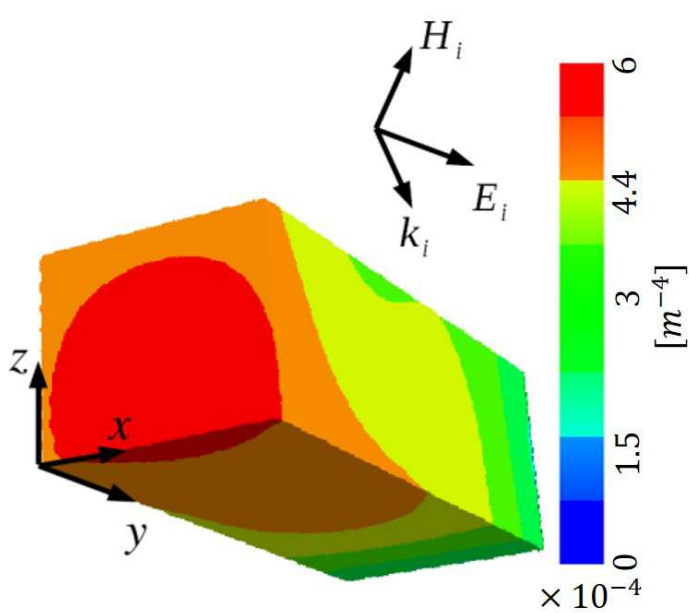

(b)

Figure 8. (a) Gold nano antenna consisting of two rectangular particles with dimensions $(a, b, c, d)=$ $(98.5,40,40,25) \mathrm{nm}$ on a glass substrate with refractive index $n_{2}=1.5$. (b) The relative sensitivity representative $\left|\frac{g}{\mathcal{J}}\right|$ over brick-1. The output functional measures the energy flux of the far-field.

other words, the output functional becomes more sensitive to the shape of $\Gamma_{s}$.

\subsection{Nano antenna consisting two rectangular blocks with displaced arms}

Now, we displace brick-2 by $\Delta x=20 \mathrm{~nm}$ in the $\hat{x}$ direction (see Figure $9 \mathrm{a}$ ). The size of the antenna, the incident field, the working frequency, the output functional, and the material of the substrate are the same as those in the previous experiment. As shown in Figure 9b, the relative sensitivity profile is also displaced in the direction $\hat{x}$. Which means the position of the antenna gap and the substrate are key elements in forming the sensitivity representative for nano antennas. 


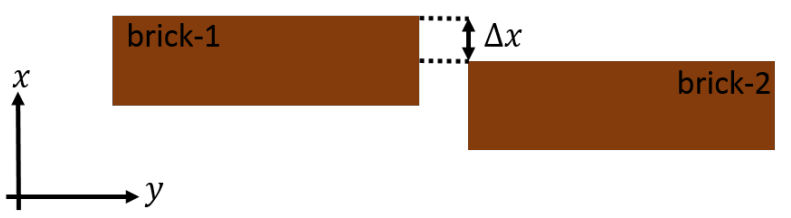

(a)

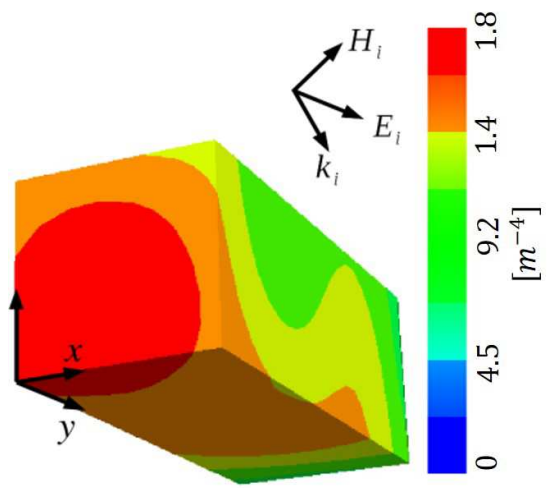

(b)

Figure 9. (a) Gold nano antenna, consisting of two rectangular blocks, with left arm displaced in $\hat{x}$ direction. The dimensions are $(a, b, c, d)=(98.5,40,40,25) \mathrm{nm}$, and $\Delta x=20 \mathrm{~nm}$. The substrate material is glass with refractive index $n_{2}=1.5$. (b) The relative sensitivity representative $\left|\frac{g}{\mathcal{J}}\right|$ over brick-1. The output functional measures the energy flux of the far-field.

\section{CONCLUSION}

Shape calculus is a technique to investigate the sensitivity of an output functional with respect to the shape of the domain. The calculation of shape gradients for 3D scattering problems gets more complicated due to the presence of vector electromagnetic fields.

We formulated shape gradients for 3D Maxwell's equation. Given that the solution of the state problem is complex valued while the shape functional is real valued, we used generalized Lagrangian approach to find $d \mathcal{J}$. In order to have a mapping which is isomorphism from the reference domain to the perturbed domain, a covariant transformation was applied.

Second order B-Splines were used as probing perturbation functions to deform the object locally. We used Hadamard-Zolesio structure theorem to obtain shape sensitivity representative based on sensitivity probings.

Several numerical experiments were conducted to investigate the shape sensitivity of different plasmonic nano antennas. Based on the obtained results, the gap area is the most sensitive part, which means small perturbations introduced in this region affects the performance of the nanoparticle significantly.

Shaping edges and corners is one of the main challenges during fabrication process. However, our results showed that the far-field functional is not highly sensitive to the shape of corners and edges. The presence of a substrate is also another issue that can affect the sensitivity of the particle. We also showed that the far-field pattern gets more sensitive to perturbations of the face touching the substrate.

\section{REFERENCES}

1. Jackson JB, Halas NJ. Surface-enhanced raman scattering on tunable plasmonic nanoparticle substrates. Proc. Nat. Acad. Sci. USA 2004; 101:17 930-17935.

2. Vosgrone T, Meixner A, Anders A, Dietz H, Sandmann G, Plieth W. Electrochemically deposited silver particles for surface enhanced raman spectroscopy. Surface Science 2005; 102:102-109.

3. Talley CE, Jackson JB, Oubre C, Grady NK, Hollars CW, Lane SM, Huser TR, Nordlander P, Halas NJ. Surfaceenhanced raman scattering from individual au nanoparticles and nanoparticle dimer substrates. Nano Lett. 2005; 5:1569-1574.

4. Nie S, Zare RN. Optical detection of single molecules. Annu. Rev. Biophys. Biomol. Struct. 1997; 26:567-596.

5. Kelly KL, Coronado E, Zhao LL, Schatz GC. The optical properties of metal nanoparticles: The influence of size, shape, and dielectric environment. Phys. Chem. B 2003; 107:668-677. 
6. Fischer H, Martin OJF. Engineering the optical response of plasmonic nanoantennas. Opt. Express 2008; 16:91449154.

7. Nordlander P, Le F. Plasmonic structure and electromagnetic field enhancements in the metallic nanoparticle-film system. Appl. Phys. B 2006; 84:35-41.

8. Delfour M, Zolesio J. Shapes and Geometries. Metrics, analysis, differential calculus, and optimization. Society for Industrial and Applied Mathematics (SIAM), 2011.

9. Sokolowski J, Zolesio J. Introduction to shape optimization. Shape sensitivity analysis. Springer-Verlag, 1992.

10. Eppler K. Boundary integral representations of second derivatives in shape in shape optimization. Discuss. Math. Differ. Incl. Control Optim. 2000; 20:63-68.

11. Eppler K. Second derivatives and sufficient optimality conditions for shape functionals. Control Cybernet. 2000; 29:485-511.

12. Simon J. Differentiation with respect to the domain in boundary value problems. Numer. Funct. Anal. Optim. 1981; 2:649-687.

13. Hiptmair R, Paganini A, Sargheini S. Comparison of approximate shape gradients. BIt Numerical mathematics 2014; electronic.

14. Cagnol J, Marmorat JP, Zolesio JP. Shape sensitivity analysis in the maxwell's equations. Shape Optimization And Optimal Design, 2001.

15. Chaulet N, Haddar H. Electromagnetic inverse shape problem for coated obstacles. Adv. Comput. Math 2015; .

16. Monk P. Finite element methods for Maxwell's equations. Clarendon Press, Oxford, 2003.

17. Hiptmair R. Finite elements in computational electromagnetism. Acta Numerica, Cambridge University Press, United Kingdom 2002; 11.

18. Delgado KK. The complex gradient operator and the cr-calculus. University of California, San Diego, CA 2009; UCSD-ECE275CG-S2009v1.0.

19. Höllig K, Hörner J. Approximation and modeling with B-splines. Society for Industrial and Applied Mathematics, Philadelphia, PA, 2013.

20. Hiptmair R, Sargheini S. Scatterers on the substrate: Far field formulas. SAM report 2015; 2015-02.

21. Johnson PB, Christy RW. Optical constants of the noble materials. Phys. Rev. B. 1972; 6(12):4370-9.

22. Smajic J, Hafner C. Plasmonic v-groove waveguides: building blucks for optical transistor. J. COMPUT. THEOR. NANOS. 2010; 7:1616-1622. 